\title{
Protective effects of Gingko biloba extract 761 on myocardial infarction via improving the viability of implanted mesenchymal stem cells in the rat heart
}

\author{
YAN-LI LIU $^{1 *}$, YAN ZHOU $^{1 *}$, LIN SUN $^{2}$, JIANG-TAO WEN $^{1}$, SHI-JIE TENG $^{1}$, LIN YANG $^{1}$ and DONG-SHU DU ${ }^{3}$ \\ ${ }^{1}$ Department of Laboratory Medicine, Lianyungang Hospital Affiliated Bengbu Medical College, Lianyungang, \\ Jiangsu 222006; ${ }^{2}$ Department of Laboratory Medicine, Lianyungang Women and Children's Health Care Hospital, Lianyungang, \\ Jiangsu 222001; ${ }^{3}$ Laboratory of Neuropharmacology and Neurotoxicology, Shanghai University, Shanghai 200444, P.R. China
}

Received January 6, 2014; Accepted January 27, 2014

DOI: $10.3892 / \mathrm{mmr} .2014 .1959$

\begin{abstract}
When introduced into the infarcted heart, bone marrow-derived mesenchymal stem cells (MSCs) prevent the heart from deleterious remodeling and improve its recovery. The aim of the present study was to investigate the effects of Ginkgo biloba extract (EGb) 761 on the infarcted myocardium microenvironment following MSC transplantation. The established rat myocardial infarction (MI) model, with implanted PKH-26 marked MSCs (1x10 cells), were randomly divided into two groups: The control group (injected with normal saline) and the EGb 761 treatment group (injected with $100 \mathrm{mg}$ / $\mathrm{kg} /$ day EGb 761). The following indices for cardiac function, including the extent of inflammation, oxidative stress, MSC apoptosis and MSC differentiation were measured 1, 2 and 7 days after treatment. The anti-inflammatory effect of EGb 761 was observed by histological examination. Compared with the respective control group, the malondialdehyde content significantly decreased and the superoxide dismutase, catalase and glutathione peroxidase activity significantly increased in the EGb761-treated groups. In addition, the apoptotic index gradually decreased $(\mathrm{P}<0.05)$ with the extension of MI time in the EGb761-treated groups compared to the respective control groups, suggesting that EGb761 exhbits anti-oxidative effects. In addition, the level of the Fas protein was positively correlated with the implanted MSC apoptotic ratio. Following 7 days of MSC transplantation with EGb 761 treatment, the expression of cTnI in PKH26-labeled MSCs was observed in the transplanted myocardium. Cardiac function, including the ejection fraction, left ventricular end-systolic pressure and
\end{abstract}

Correspondence to: $\mathrm{Dr}$ Dong-Shu Du, Laboratory of Neuropharmacology and Neurotoxicology, Shanghai University, No. 99 Shangda Road, Shanghai 200444, P.R. China

E-mail: sdhzdds@163.com

*Contributed equally

Key words: Gingko biloba extract 761, mesenchymal stem cells, myocardial infarction dp/dtmax significantly increased, and the left ventricular end diastolic diameters, left ventricular end-diastolic volumes and left ventricular end-diastolic pressure significantly decreased $(\mathrm{P}<0.05$, vs. the control group). The results demonstrated that EGb 761 is important in improving cardiac function and the infarcted myocardium microenvironment. The present study indicated that the protective effects of EGb 761 on the infarcted myocardium may be mediated by improving the viability and the differentiation of the implanted MSCs into cardiomyocytes.

\section{Introduction}

Myocardial infarction (MI) leads to the permanent loss of cardiomyocytes, scar formation and the absence of the endogenous repair mechanism which leads to heart failure (1). Bone marrow-derived mesenchymal stem cells (MSCs) are self-renewing, multipotent adult cells (2), which are able to differentiate into osteoblasts, chondrocytes (3), astrocytes (4), neurons (5), endothelial cells (6), skeletal muscle cells (7), vascular smooth muscle cells (6) and cardiomyocytes in vitro (8) and in vivo (9-10). Animal and clinical studies have provided evidence that cell therapy with MSCs is able to improve cardiac function potentially via angiogenesis and myogenesis following MI (11-12). However, the clinical exploitation of MSC transplantation is hampered by their poor viability in the diseased myocardium post-transplantation. For instance, the majority of MSCs injected into the left ventricle of the adult murine heart died within 1 week during the injection period (9). This reflects that the ischemic microenvironment of the infarcted myocardium was not conducive of MSC survival. Furthermore, a large number of studies have demonstrated that increasing the differentiation of implanted MSCs into cardiomyocytes was able to promote heart function during the acute phase of MI (13-15). Therefore, promoting the survival of implanted MSCs and improving the differentiation of implanted MSCs into cardiomyocytes following transplantation is critical for successful cellular therapy.

The extract of Ginkgo biloba (EGb) leaves has been applied as a traditional Chinese medicine for numerous years. 
At present, the extract of the standardized G. biloba leaf with well-defined components, labeled EGb 761, which contains $24 \%$ ginkgo-flavone glycosides (e.g. kaempferol, quercetin and isorhamnetin derivatives) and 6\% terpenoid (e.g. ginkgolides A, B, C, J and bilobalide), has been developed and is extensively consumed as a dietary supplement and a herbal remedy (16). Numerous studies have verified that EGb 761 protects the cell from a variety of extrinsic toxic stimuli, which induce damage in different experimental disease models (17-20). Previous studies have reported that EGb 761 mediates its protective effects against doxorubicin-induced cardiac injury through antioxidant, anti-inflammatory and antiapoptotic mechanisms. Furthermore, it may induce neuronal differentiation of cultured PC12 cells and neuroblast differentiation in the mouse hippocampal dentate gyrus, and promote the differentiation of human umbilical cord-derived mesenchymal stem cells (21-24). However, it is unclear whether EGb 761 is able to improve the cardiac performance of infarcted hearts through increasing the viability of implanted MSCs and improving the differentiation of implanted MSCs into cardiomyocytes following cellular therapy. The present study aimed to investigate the roles and the potential mechanisms of EGb 761 on implanted MSCs in the infarcted myocardium, and to provide experimental evidence for the potential application of EGb 761 for cellular therapy during ischemic heart disease.

\section{Materials and methods}

Reagents. The reagents and detection kits used were as follows: Low-Dulbecco's modified Eagle's medium (L-DMEM), trypsin-EDTA, fetal bovine serum, penicillin and streptomycin were purchased from Gibco-BRL (Carlsbad, CA, USA); malondialdehyde (MDA) and superoxide dismutase (SOD)assay kits were obtained from Nanjing Jiancheng Biochemical Institute (Nanjing, Jiangsu, China); EGb 761 was obtained from Invitrogen Life Technologies (Karlsruhe, Germany); the In situ Cell Death Detection kit was purchased from Nanjing KeyGen Biochemical Institute (Nanjing, China); Fas and cTnI antibodies were obtained from Antibody Design Labs (San Diego, CA, USA); and IRDye $800 \mathrm{CW}$ Donkey anti-rabbit IgG, IRDye $800 \mathrm{CW}$ Donkey anti-mouse IgG and IRDye 680 Donkey anti-goat IgG were purchased from Li-Cor Biosciences, (Lincoln, NE, USA). Unless stated otherwise, all other chemicals were purchased from Sigma (St. Louis, MO, USA).

Animals. Sprague-Dawley rats weighing $250 \pm 15 \mathrm{~g}$ were obtained from the Experimental Animal Center of Shanghai Medical College (Shanghai, China). All animals were housed in a light-controlled room with a $12 \mathrm{~h}$ light/dark cycle and were allowed access to food and water ad libitum. Experimental protocols and animal care methods were approved by the Experimental Animal Research Committee of Fudan University (Shanghai, China).

Cell culture. The MSCs were obtained from bone marrow aspirates of femurs and tibias from rats using a modified method originally described by Xie et al (25). The marrow pellet was washed in phosphate-buffered saline (PBS), centrifuged at 2,000 $\mathrm{x}$ g for $18 \mathrm{~min}$ and then resuspended in L-DMEM. Nucleated cells were isolated with a density gradient centrifugation (Ficoll or Paque, GE Healthcare Life Sciences, Schenectady, NY, USA), then introduced into a $25-\mathrm{cm}^{2}$ flask and cultured (at a density of $5 \times 10^{7}$ cell $/ \mathrm{ml}$ ) at $37^{\circ} \mathrm{C}$ in humidified air with $5 \% \mathrm{CO}_{2}$ in L-DMEM containing $15 \%$ fetal calf serum (FCS), penicillin (100 U/ml) and streptomycin $(100 \mathrm{mg} / \mathrm{ml})$. The medium was changed to remove non-adherent cells $72 \mathrm{~h}$ after seeding and every 3 days for $\sim 10$ days.

The primary cultured cells were replated into two new flasks when the MSCs had grown to $~ 80 \%$ confluence. For subculture, the cells were resuspended with $0.25 \%$ trypsin $+0.02 \%$ EDTA and passaged at a ratio of 1:2 plates. For the four passages, homogeneous MSCs devoid of hematopoietic cells were used for the experiments.

The cultured cells were initially identified by fluorescence-activated cell sorting (FACS) analysis prior to the experiments. Briefly, MSCs were trypsinized and washed with $4 \%$ FCS buffer in PBS. Then, $\sim 10^{6} / 100 \mu$ l cells were incubated at $4^{\circ} \mathrm{C}$ for $30 \mathrm{~min}$ with $20 \mu \mathrm{l}$ antibodies, i.e phycoerythrin-conjugated anti-rat CD14, CD90 and CD45 (AbD Serotec, Düsseldorf, Germany), fluorescein isothiocyanate (FITC)-conjugated anti-rat CD105 and CD44 (BioLegend, San Diego, CA, USA) and FITC-conjugated anti-rat CD34 antibodies (Santa Cruz Biotechnology, Inc, Santa Cruz, CA, USA). A normal mouse IgG was considered as the negative isotype control. The cells were washed twice with PBS and were then labeled with rabbit or mouse-FITC conjugated IgG for $20 \mathrm{~min}$ in a dark environment at room temperature. Flow cytometric analyses were performed on a FACS Calibur system using Cell Quest TM software (Becton-Dickinson, Franklin Lakes, NJ, USA). In total, 10,000 events were recorded for each sample.

MI animal model and MSC transplantation. The rats underwent myocardial ischemia by occlusion of the left coronary artery. Briefly, the rats were anesthetized by intraperitoneal injection of sodium pentobarbital $(45 \mathrm{mg} /$ $\mathrm{kg}$ ). The animal hearts were exposed by a left thoracotomy. Ligation of the left anterior descending (LAD) artery was performed 1-2 mm distal to the line between the left border of the pulmonary conus and the right border of the left atrial appendage. MI was confirmed by electrocardiogram (ECG; ADI Instruments, Houston, TX, USA). A final $200 \mu 1$ suspension containing a total of $1 \times 10^{5}$ MSCs labeled with PKH-26 red fluorescent cell tracker dye (Red Fluorescent Cell Linker Kit; Sigma) was then transplanted into four sites around the ischemic myocardium marginal zone of the left ventricle via intramyocardial injection. The animal chest cavity was closed following transplantation of MSCs. The rats were left to recover in a temperature-controlled chamber until they resumed full alertness and mobility, at which point they were returned to their cages.

The animals were randomly divided into two groups. The control group and the EGb 761-treated groups. In the control group, following MSC transplantation, $3 \mathrm{ml}$ normal sodium $(0.9 \% \mathrm{NaCl})$ was injected intraperitoneally $(n=18)$ and in the EGb 761-treated groups, following MSC (1x10 $)$ transplantation, $100 \mathrm{mg} / \mathrm{kg} /$ day EGb 761 (3 ml) was injected 
intraperitoneally $(n=18)$. For each animal, the total quantity of normal sodium and EGb 761 was divided into two intraperitoneal injections per day, respectively. Following 1,2 and 7 days of MSC transplantation and EGb 761 treatment, the animal hearts from each group (six animals per group) were harvested under inhalation anesthesia, respectively, and fixed with $4 \%$ neutral formaldehyde for further analysis.

Measurement of cardiac function. Following 1, 2 and 7 days of normal sodium and EGb 761 treatment, the cardiac function of rats was assessed by a multichannel physiological recorder (Jinjiang Tongyong Industry Co., Ltd., Sichuan, China). Briefly, after obtaining the body weight of each animal, animals were sedated $(100 \mathrm{mg} / \mathrm{kg}$ ketamine and $1.5 \mathrm{mg} / \mathrm{kg}$ xylazine) and a transducer (Jinjiang Tongyong Industry Co., Ltd., Chengdu, China) was applied to the left hemithorax. The heart was visualized using a 2-dimensional mode with the axial view of the left ventricle. Next, through the right carotid artery, a cannula was inserted into the left ventricle. The ejection fraction (EF), left ventricular end-diastolic volumes (LVEDV), left ventricular end-diastolic pressure (LVEDP), left ventricular $\pm \mathrm{dp} / \mathrm{dt}$ (maximum rate of pressure rise), left ventricular end diastolic diameters (LVDd), fractional shortening (FS) and left ventricular end-systolic pressure (LVESP) were calculated by the standard formulas of ECToolbox software (Emory University, Atlanta, GA, USA).

Histopathological examination. Following 1,2 and 7 days of normal sodium and EGb 761 treatment, each group of animals were sacrificed by high dose anesthesia $(45 \mathrm{mg} / \mathrm{kg}$, respectively, and perfused with $0.9 \%$ saline followed by $4 \%$ paraformaldehyde through a needle inserted into the left ventricle of the rat through the left atrium, for in situ perfusion fixation. Myocardial tissues in the left ventricle of sacrificed rats were collected and fixed in $4 \%$ pre-cooled paraformaldehyde for $72 \mathrm{~h}$ and embedded in paraffin for histological studies. Paraffin-embedded tissues were sectioned into $5-\mu \mathrm{m}$ thick slices. The sections were stained for hematoxylin and eosin (H\&E) according to the manufacturer's instructions (Beyotime Biochemical Institute, Shanghai, China). Images were visualized under an optical microscope at x100 and x400 magnifications.

Determination of reactive oxygen species production. Following 1,2 and 7 days of normal sodium and EGb 761 treatment, each group of animals was sacrificed using high-dose anesthesia (sodium pentobarbital, $45 \mathrm{mg} / \mathrm{kg}$ ), respectively. The hearts of the animals were then removed and opened along the greater curvature and washed with PBS. The homogenate was centrifuged (10,000 x g for $15 \mathrm{~min})$ and then the supernatant was subjected to glutathione peroxidase (GSH-Px), catalase (CAT) and superoxide dismutase (SOD) activity, and malondialdehyde (MDA) content assays, according to the manufacturer's instructions.

Terminal dUTP nick-end labeling (TUNEL) assay. The cryosections (40- $\mu \mathrm{m}$ thick) from animal myocardial samples were stained with an in situ cell death detection kit (Kaiji Biological Technology development Co., Ltd., Nanjing, China) according to the manufacturer's instructions, and then the stained cryo- sections were observed with a confocal microscope (Leica TCS SP8 MP, Leica, Wetzlar, Germany). The apoptotic index (AI) was determined using the following formula: $\mathrm{AI}=$ number of TUNEL-positive cells / total transplanted cells pre-labeled with PKH-26 x 100.

Western blot analysis. The infarcted heart tissues were lysed in Tris-HCl buffer $(0.05 \mathrm{~mol} / \mathrm{l})$ containing $0.15 \mathrm{~mol} / \mathrm{l}$ $\mathrm{NaCl}, 0.02 \% \mathrm{NaN}_{3}, 0.1 \%$ SDS, $1 \%$ nonidet P (NP-40), $11 \mu \mathrm{g} / \mathrm{ml}$ aprotinin and $0.1 \mathrm{~mol} / 1$ phenylmethyl sulfonylfluoride $(\mathrm{pH} 8.0)$. The expression of the Fas protein was determined by immunoblotting. Briefly, the protein content of the supernatant prepared from cultured cells was quantified by Lowry's method. A $100 \mu \mathrm{g}$ sample of total protein was separated by $10 \%$ SDS-PAGE and then transferred onto a polyvinylidene fluoride membrane (pore size, $0.45 \mu \mathrm{m}$; $3.0 \mathrm{~mA} / \mathrm{cm}^{2}$ for $40 \mathrm{~min}$ ). Following inhibition with $5 \%$ non-fat milk powder/PBS containing 5\% Tween-20 (PBS-T) for $3 \mathrm{~h}$ at room temperature, the membranes were incubated with the following antibodies: Mouse anti-fas and rabbit anti-GAPDH. The membranes were probed with the primary antibodies overnight at $4^{\circ} \mathrm{C}$. Then, IRDye-conjugated secondary antibodies (IRDye donkey anti-mouse IgG and IRDye donkey anti-rabbit $\operatorname{IgG} ; 1: 100)$ were added for $2 \mathrm{~h}$ and subsequently scanned by the Odyssey Infrared Imaging system (Li-Cor Biosciences). Specific densitometry values for the bands were calculated by normalization to GAPDH.

Differentiation of implanted cells. Animals were sacrificed using high-dose anesthesia (sodium pentobarbital, $45 \mathrm{mg} / \mathrm{kg}$ ). The hearts were quickly harvested and tissues from the free wall of the left ventricle, including the infarct and peri-infarct regions were then embedded in optimal cutting temperature tissue-freezing medium. The frozen sections (40- $\mu \mathrm{m}$ thick) of the left ventricular samples were prepared for identification of implanted cells. Briefly, the mounted frozen sections on glass slides were fixed with methanol at $-20^{\circ} \mathrm{C}$ for $20 \mathrm{~min}$. Following washing three times with PBS, the sections were incubated with blocking buffer (1\% donkey serum, $1 \%$ bovine serum albumin and $0.2 \%$ Tween-20 in PBS) for $30 \mathrm{~min}$ at room temperature and then with the mouse anti-rat cTnI antibodies in blocking buffer for $1 \mathrm{~h}$ at room temperature. The sections were then washed three times in PBS, followed by incubation with FITC-conjugated goat anti-mouse IgG second antibody for $1 \mathrm{~h}$ at room temperature. Following washing three times with PBS, the stained cryosections were observed under a confocal microscope (Leica).

Statistical analysis. All data are expressed as the mean \pm standard error of the mean and analyzed by analysis of variance and a Student-Newman-Keuls test using SPSS 18.0 software (SPSS Inc., Chicago, IL, USA). P $<0.05$ was considered to indicate a statistically significant difference.

\section{Results}

Expression of surface markers of MSCs. Consistent with previous studies $(25,26)$, the cultured MSCs expressed typical mesenchymal markers, including CD44, CD90 and CD105. However, the surface markers of hematopoietic stem cells 
Table I. EGb 761 ameliorates cardiac function.

\begin{tabular}{|c|c|c|c|c|c|c|}
\hline \multirow[b]{2}{*}{ Group } & \multicolumn{2}{|c|}{1 day } & \multicolumn{2}{|c|}{2 days } & \multicolumn{2}{|c|}{7 days } \\
\hline & Control & EGb 761 & Control & EGb 761 & Control & EGb 761 \\
\hline $\mathrm{EF}(\%)$ & $72.32 \pm 1.46$ & $75.29 \pm 2.43^{\mathrm{a}}$ & $70.34 \pm 0.51^{\mathrm{b}}$ & $78.53 \pm 0.95^{\mathrm{a}, \mathrm{b}}$ & $68.57 \pm 0.43^{c}$ & $83.66 \pm 1.57^{\mathrm{a}, \mathrm{c}}$ \\
\hline FS $(\%)$ & $40.20 \pm 0.56$ & $43.32 \pm 1.82^{\mathrm{a}}$ & $35.43 \pm 1.43^{\mathrm{b}}$ & $47.64 \pm 1.55^{\mathrm{a}, \mathrm{b}}$ & $30.12 \pm 2.00^{c}$ & $53.00 \pm 2.51^{\mathrm{a}, \mathrm{c}}$ \\
\hline LVDd (cm) & $0.62 \pm 0.04$ & $0.57 \pm 0.05^{\mathrm{a}}$ & $0.67 \pm 0.02^{\mathrm{b}}$ & $0.53 \pm 0.08^{\mathrm{a}, \mathrm{b}}$ & $0.73 \pm 0.06^{\mathrm{c}}$ & $0.48 \pm 0.03^{\mathrm{a}, \mathrm{c}}$ \\
\hline LVEDV (ml) & $0.310 \pm 0.021$ & $0.300 \pm 0.034^{\mathrm{a}}$ & $0.315 \pm 0.038^{\mathrm{b}}$ & $0.290 \pm 0.033^{\mathrm{a}, \mathrm{b}}$ & $0.320 \pm 0.030^{c}$ & $0.280 \pm 0.040^{\mathrm{a}, \mathrm{c}}$ \\
\hline LVESP (mmHg) & $105.40 \pm 3.28$ & $115.40 \pm 3.38^{\mathrm{a}}$ & $98.40 \pm 3.16^{\mathrm{b}}$ & $125.40 \pm 3.68^{\mathrm{a}, \mathrm{b}}$ & $92.10 \pm 2.32^{\mathrm{c}}$ & $135.400 \pm 3.41^{\mathrm{a}, \mathrm{c}}$ \\
\hline LVEDP (mmHg) & $9.300 \pm 0.31$ & $7.300 \pm 0.22^{\mathrm{a}}$ & $11.300 \pm 0.28^{\mathrm{b}}$ & $6.306 \pm 0.21^{\mathrm{a}, \mathrm{b}}$ & $12.320 \pm 0.31^{\mathrm{c}}$ & $5.300 \pm 0.18^{\mathrm{a}, \mathrm{c}}$ \\
\hline $\mathrm{dp} / \mathrm{dtmax}(\mathrm{mmHg} / \mathrm{sec})$ & $6560.5 \pm 133.5$ & $7260.5 \pm 135.9^{a}$ & $5860.5 \pm 115.5^{b}$ & $7950.4 \pm 136.6^{\mathrm{a}, \mathrm{b}}$ & $4860.3 \pm 210.3^{c}$ & $8500.0 \pm 234.3^{\mathrm{a}, \mathrm{c}}$ \\
\hline
\end{tabular}

Data are shown as the mean \pm standard error of the mean $(n=8)$. EF, ejection fraction; FS, fractional shortening; LVDd, left ventricular end diastolic diameters; LVEDV, left ventricular end-diastolic volumes; LVESP, left ventricular end-systolic pressure; LVEDP, left ventricular end-diastolic pressure; dp/dtmax, left ventricular maximum rate of pressure rise. ${ }^{\mathrm{P}} \mathrm{P}<0.05$, vs. the respective control group, ${ }^{\text {b }} \mathrm{P}<0.05$, vs. the 1 day group; ${ }^{\mathrm{P}}<0.05$, vs. the 2 days group. EGb, extract of Ginkgo biloba.
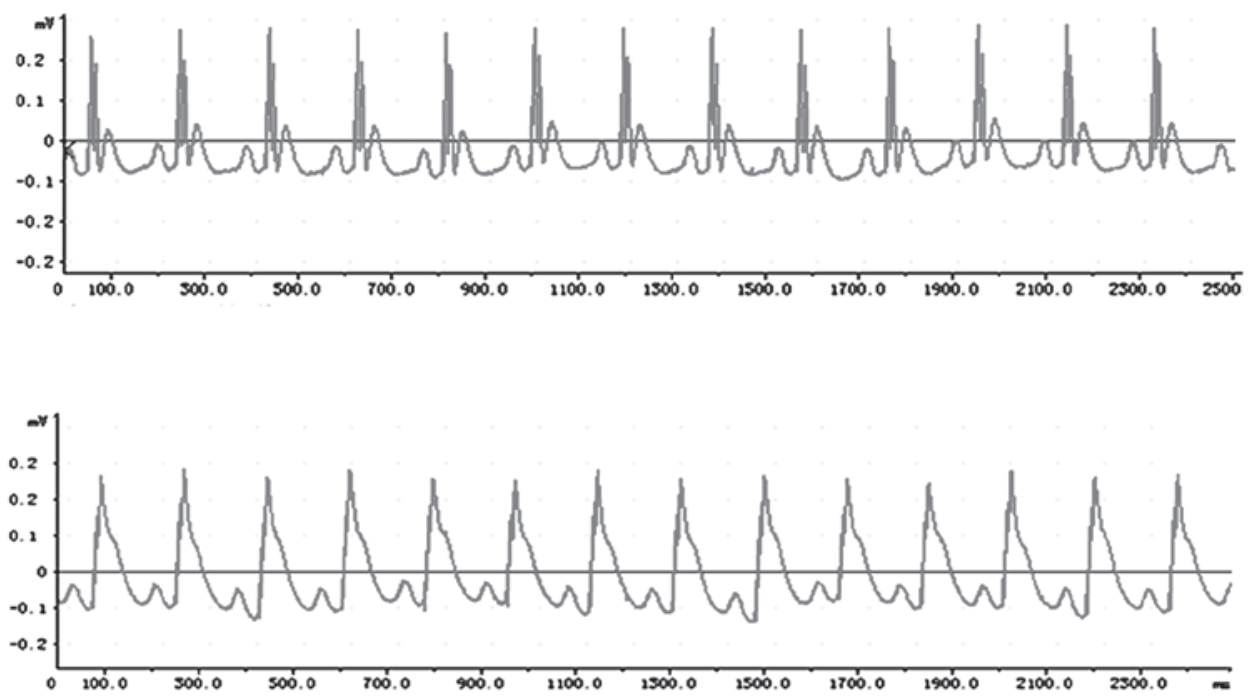

Figure 1. ECG recordings in rats. (A) surface ECG from a normal rat that had not undergone ligation of athe LAD coronary artery; (B) typical ECG pattern produced during the ligation procedure, indicating MI-induced deep S wave, diminished R-wave amplitude and marked ST segment elevation. ECG, electrocardiogram; LAD, left anterior descending; MI, myocardial infarction.

(CD45), macrophages (CD14) and lymphocytes (CD34) were not expressed (data not shown).

Establishment of the MI model. Compared with the ECG trace from a normal rat that had not undergone ligation of the LAD coronary artery (Fig. 1A), a deep S wave, R-wave elimination ST segment elevation was observed on the ECG from a rat that had undergone ligation of LAD coronary artery (Fig. 1B).

Amelioration of cardiac function by EGb 761 treatment. The parameters of echocardiography and hemodynamics were analyzed. Compared with the respective control group, FS, EF, LVESP and dp/dtmax significantly increased, and LVDd, LVEDV and LVEDP significantly decreased following EGb 761 treatment $(\mathrm{P}<0.05$ vs. the control group; Table I).
EGb 761 reduces $M I$-induced heart inflammation. H\&E staining images demonstrated that MI elicited typical cardiac histopathological changes in the myocardium, which were manifested as the presence of mononuclear cells, fragments of necrotic myocardial fibers, eosinophils, polymorphonuclear neutrophils and macrophagocytes. Compared with the respective control group, EGb 761 significantly reduced the number of infiltrated inflammatory cells in the infarcted myocardium following 1,2 and 7 days of MSC transplantation (Fig. 2).

Anti-oxidative effects of EGb 761 on the heart. The effects of EGb 761 on the activity of the antioxidant enzymes SOD, CAT and GSH-Px, and the level of MDA in the heart tissues of rats are shown in Fig. 3. The results indicated that MDA content (a product of lipid peroxidation) significantly decreased 


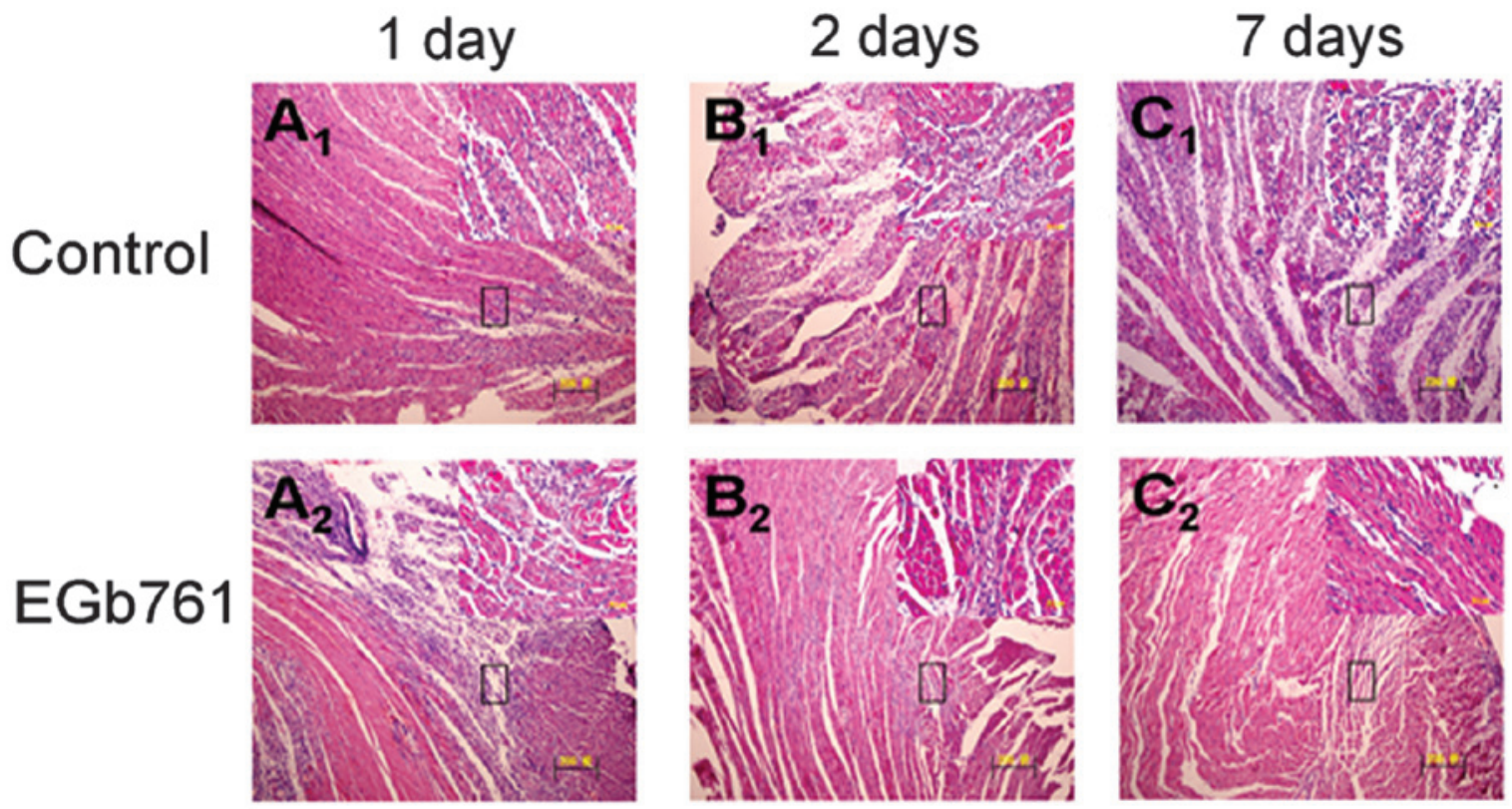

Figure 2. Pathological structures of the heart by hematoxylin and eosin staining (magnification, $x 100$ and magnification, $x 400)$. $\left(A_{1}-C_{1}\right)$ Control group; $\left(\mathrm{A}_{2}-\mathrm{C}_{2}\right)$ EGb 761-treated groups; $\left(\mathrm{A}_{1}\right.$ and $\left.\mathrm{A}_{2}\right)$ Pathological images of the heart following 1 day of $\mathrm{MI} ;\left(\mathrm{B}_{1}\right.$ and $\left.\mathrm{B}_{2}\right)$ Pathological images of the heart following 2 days of MI; $\left(\mathrm{C}_{1}\right.$ and $\left.\mathrm{C}_{2}\right)$ Pathological images of the heart following 7 days of MI. MI, myocardial infarction; EGb, extract of Ginkgo biloba.

A
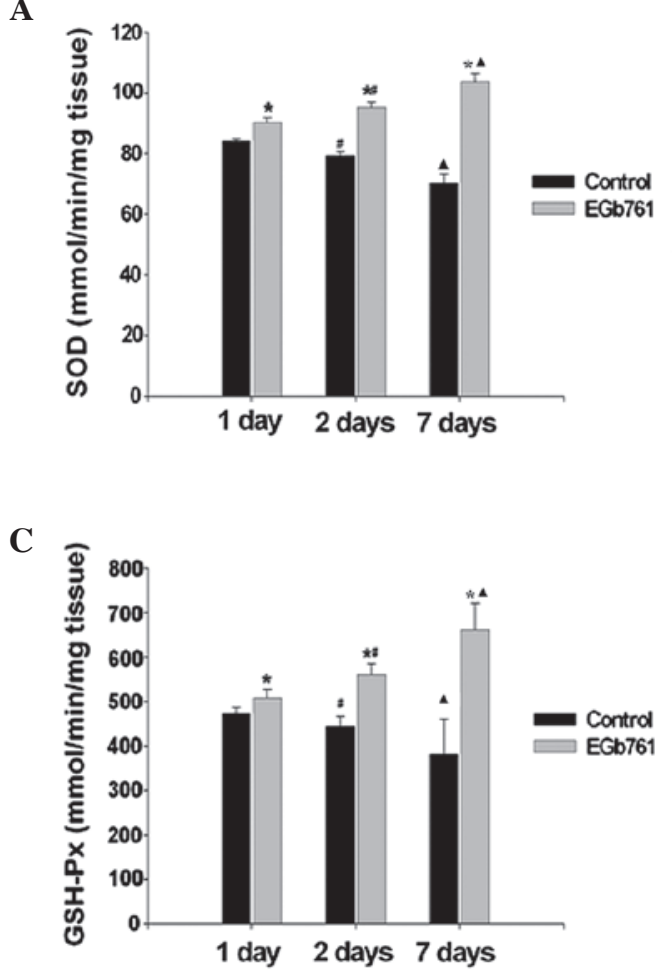

B
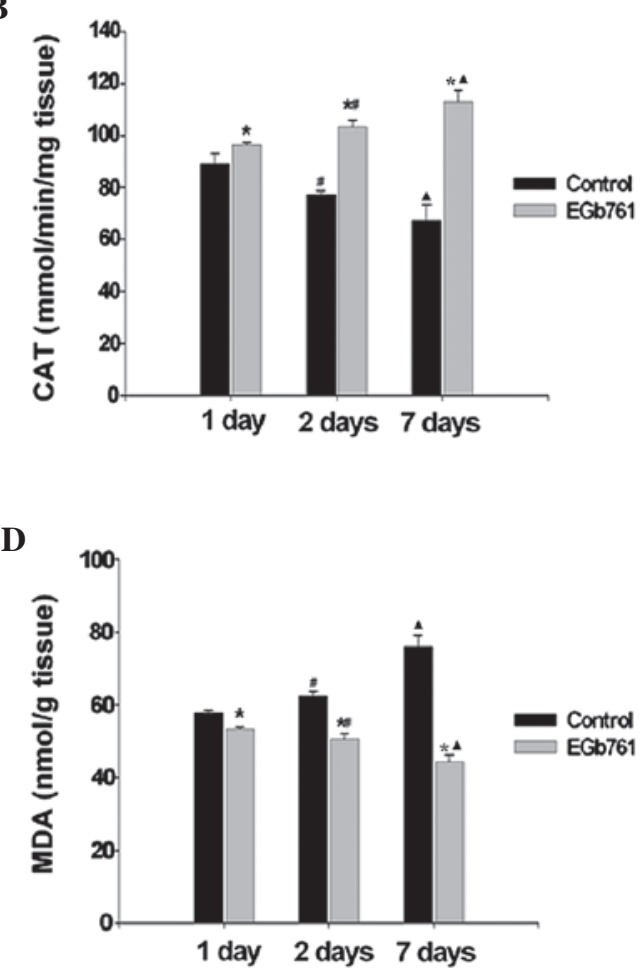

Figure 3. Effects of EGb 761 on MI-induced oxidative stress. Activity of (A) SOD, (B) CAT, (C) GSH-Px and (D) MDA are presented as histograms. "P<0.05, vs. the respective control group, ${ }^{*} \mathrm{P}<0.05$, vs. the 1 day group. ${ }^{\wedge} \mathrm{P}<0.05$, vs. the 2 days group. $(\mathrm{n}=6)$. MI, myocardial infarction; SOD, superoxide dismutase; CAT, catalase; GSH-Px, glutathione peroxidase; MDA, malonyldialdehyde; EGb, extract of Ginkgo biloba.

and the SOD, CAT and GSH-Px activity increased following EGb 761 treatment. With the extension of time of MI, MDA content gradually increased and the activity of SOD, CAT and GSH-Px gradually decreased in the control group. Compared with the respective control group, the activity of SOD, CAT and GSH-Px gradually increased and MDA content gradually decreased in the EGb 761 treatment group $(\mathrm{P}<0.05)$.

Antagonism of EGb 761 on MI-induced MSC apoptosis. Compared with the respective control group, the AI in the 
A

A
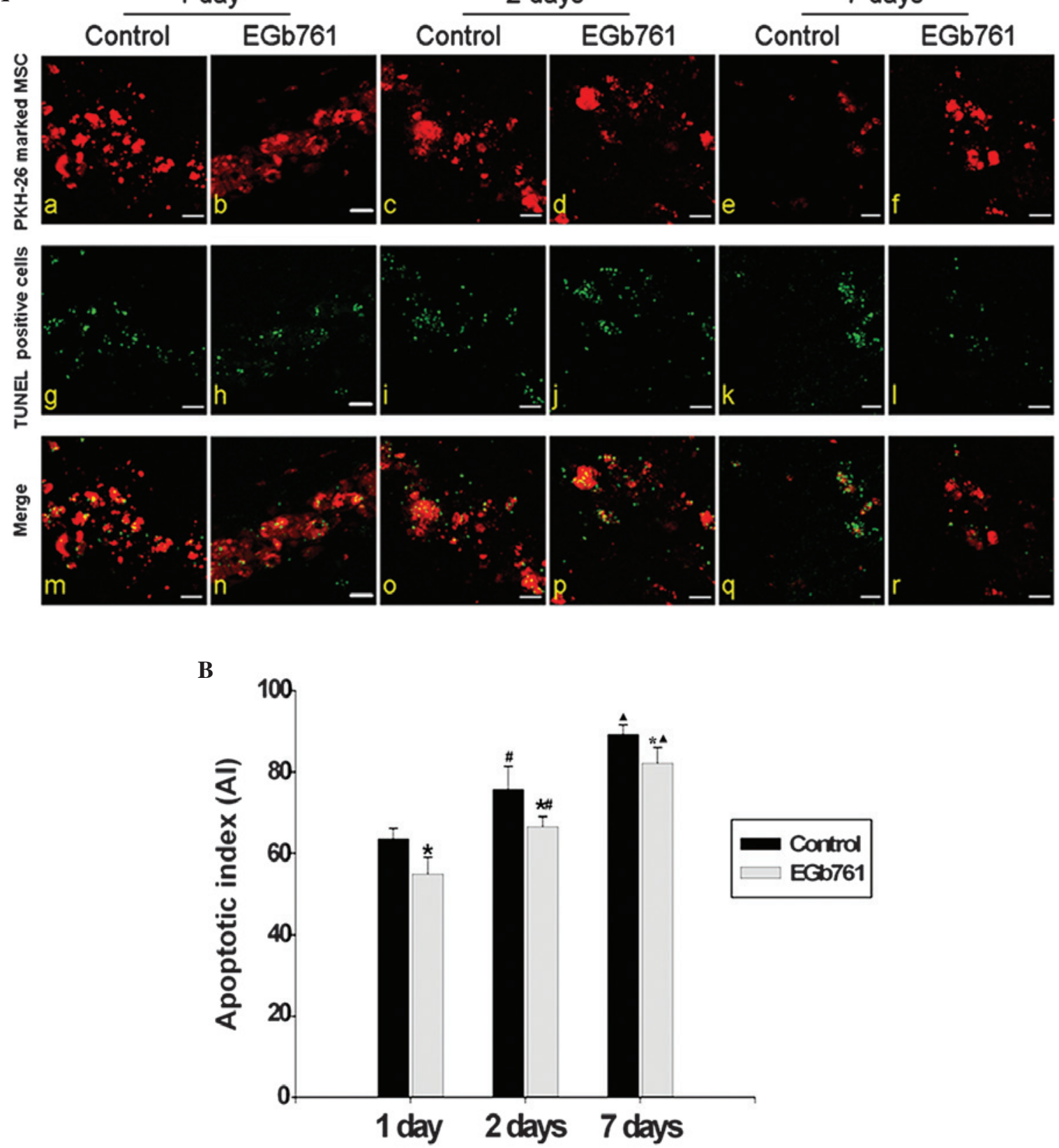

Figure 4. Effect of EGb 761 on the apoptosis of transplanted MSCs. (A) MSCs labeled with PKH-26 (red) were transplanted into the rat ischemic myocardium. Cellular apoptosis was determined using TUNEL-positive staining (green). The co-labeling of PKH-26 (red) and TUNEL (green) indicated cell apoptosis following MSC transplantation (n=6). (Aa-f) The transplanted cells identified by PKH-26-positive staining (red). (Ag-i) The apoptotic cells identified with TUNEL-positive staining (green). (Am-r) Merged figures show the apoptosis of the grafted MSCs co-labeled with PKH-26 (red) and TUNEL (green; scale bar, $10 \mu \mathrm{m}$ ). (Aa, c, e, g, i, k, m, o and q) Control group; (Ab, d, f, h, j, 1, n, p and r) EGb 761-treated groups; (Aa, b, g, h, m and $\mathrm{n}$ ) Apoptotic images of transplanted MSCs following 1 day of MI; (Ac, d, i, j, o and p) Apoptotic images of transplanted MSCs following 2 days of MI; (Ae, $\mathrm{f}, \mathrm{k}, 1, \mathrm{q}$ and $\mathrm{r}$ ) Apoptotic images of transplanted MSCs following 7 days of MI. (B) Apoptotic indexes are presented as histograms. "P $<0.05$, vs. the respective control group. There were clear differences between the 1 day group and the 2 day group $\left({ }^{*} \mathrm{P}<0.05\right)$, and between the 2 day group and the 7 day group $\left({ }^{\wedge} \mathrm{P}<0.05\right)$. $\mathrm{n}=6$. MSCs, bone marrow-derived mesenchymal stem cells; TUNEL, terminal dUTP nick-end labeling; MI, myocardial infarction; EGb, extract of Ginkgo biloba.

EGb 761 treatment group was significantly decreased $(\mathrm{P}<0.05)$. With the extension of MI time, the AI gradually increased in the control groups and the EGb761 treatment groups $(\mathrm{P}<0.05$; Fig. 4A and B).

Expression of Fas protein. As shown in Fig. 5, western blotting indicated that Fas protein was upregulated gradually and reached a high level following 7 days of normal sodium treatment in the control group, whereas the Fas protein was downregulated gradually in the EGb 761 treatment group. Compared with the respective control group, the level of Fas expression was significantly decreased $(\mathrm{P}<0.05)$.

Differentiation of MSCs following transplantation. To detect whether implanted MSCs differentiated into cardiomyocytes, the cTnI-expression in the PKH26-labeled 

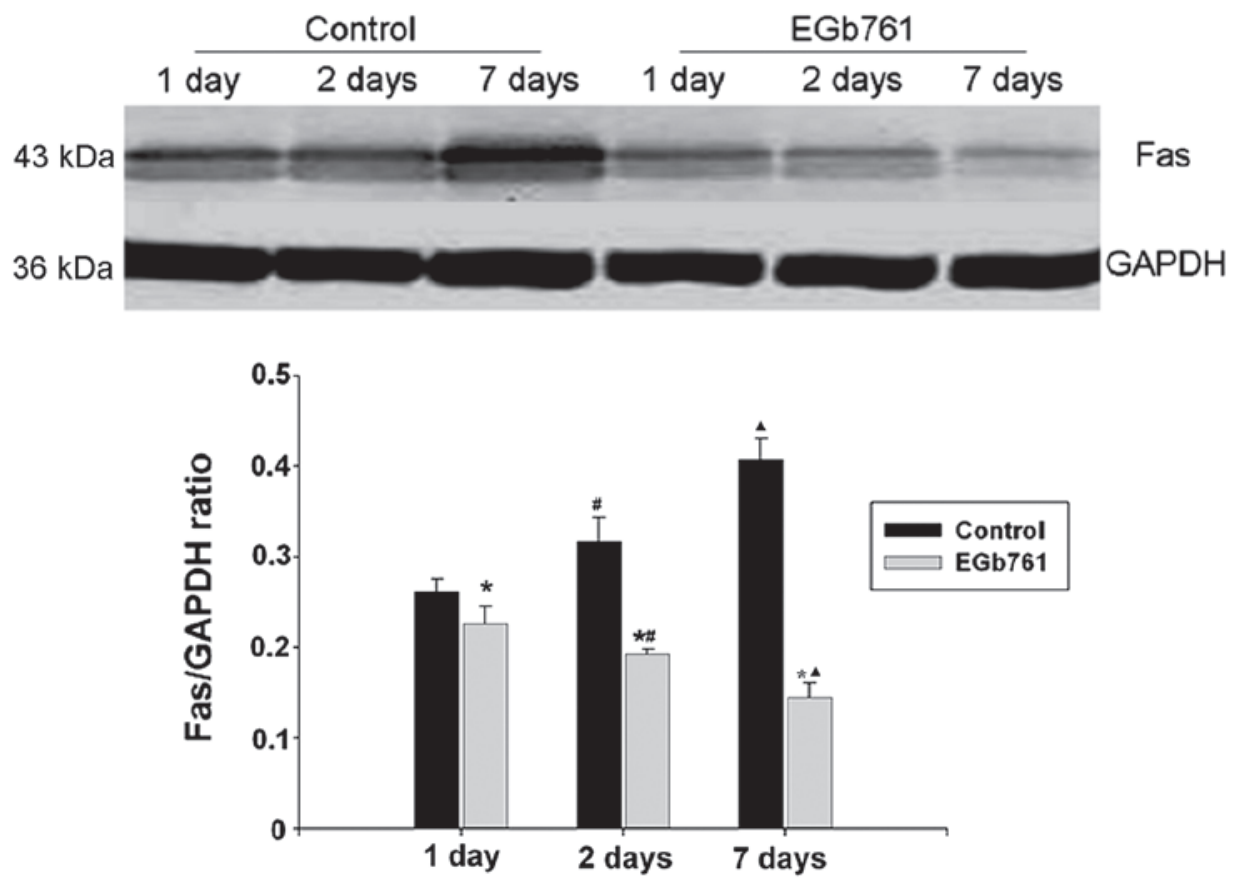

Figure 5. Western blotting of Fas protein. Expression of Fas protein in the EGb 761 treatment groups was significantly lower than that in the control group. The level of Fas expression gradually increased in the control group, whereas it decreased gradually in the EGb 761 treatment group. "P<0.05, vs. the respective control group, ${ }^{*} \mathrm{P}<0.05$, vs. the 1 day group. ${ }^{\wedge} \mathrm{P}<0.05$, vs. the 2 days group. $(\mathrm{n}=6) . \mathrm{EGb}$, extract of Ginkgo biloba.

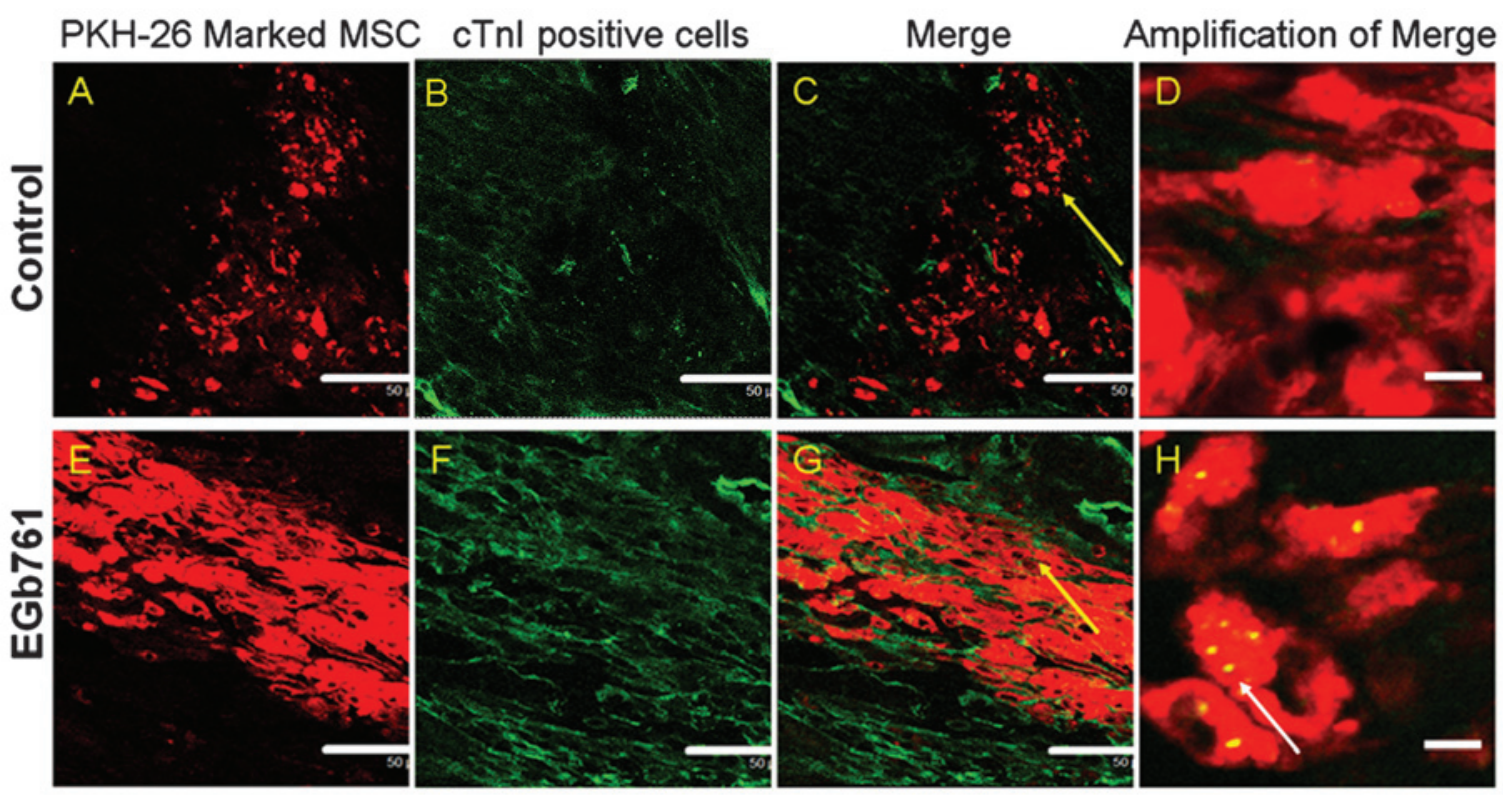

Figure 6. Cardiac differentiation of PKH 26-labeled MSCs in the transplanted myocardium following 7 days of MSC-transplantation. (A and E) MSCs labeled with PKH-26 (red) were transplanted into rat ischemic myocardium; (B and F) cell differentiation was determined with cTnI-positive staining (green); (C-D and G-H) co-labeling of PKH-26 (red) and cTnI (green) indicated cell differentiation following MSC-transplantation. D and H (Scale bar=10 $\mu \mathrm{m})$ are an amplification of C and G, respectively. The white arrow indicates the stained positive cells. Scale bar, $100 \mu \mathrm{m}$ in figure A, B, C, E, F and G. MSCs, bone marrow-derived mesenchymal stem cells; EGb, extract of Ginkgo biloba.

MSCs of the transplanted myocardium was assessed using immunohistological staining following 7 days of MSC transplantation. The results demonstrated that, in the control group, the muscle-specific markers, including cTnI staining were not expressed on the PKH26-positive cells as observed by confocal microscopy (Fig. 6A-D). Conversely, co-localization expression of cTnI and PKH26 was observed in the MSCs of the EGb 761 treatment group (Fig. 6E-H).

\section{Discussion}

MSC therapy prevents disadvantaged remodeling of heart function after MI and improves recovery when introduced into the infarcted heart; however, further promotion of the differentiation of MSCs in the cardiac scar tissue is required. The present study examined a novel strategy to promote the therapeutic effects of MSCs within the ischemic heart and 
improve cardiac function under pathological conditions. The present study indicated that EGb 761 is able to improve deteriorated cardiac function, which partially contributes to the protective effect of EGb 761 on the grafted MSCs against MI-induced apoptosis and its promotion for the differentiation of MSCs into myocardial cells. Several previous studies have demonstrated that EGb 761 reduces extrinsic damage and anti-oxidative stress, which induced the apoptosis of cardiomyocytes $(26,27)$. The present study demonstrated new evidence that EGb 761 promotes heart function recovery through enhancing implanted cell survival and differentiation in the ischemic myocardium.

Cardiac inflammation is associated with left ventricular remodeling in the MI model. Previous studies have demonstrated that autologous or allogeneic MSCs markedly suppress T-lymphocyte proliferation in the infarcted myocardium $(28,29)$. Furthermore, previous evidence suggests that the transplantation of MSCs attenuated histiocytic infiltration in a rat model of acute myocarditis (30). However, inflammation remains one of the main reasons for the low survival rate of transplanted cells in the infarcted myocardium. The present study revealed that EGb 761 significantly reduced the number of infiltrated inflammatory cells in the infarcted myocardium, which was consistent with other studies demonstrating the anti-inflammatory property of EGb 761 in various animal models $(31,32)$.

Consistent with other studies of the anti-oxidative effect of EGb 761 in vivo and in vitro $(33,34)$, the present study also demonstrated that EGb 761 had an anti-oxidative effect on the infarcted myocardium. In the present study, its effects on the reduction of MDA content and the enhancement of the activity of antioxidant enzymes SOD, CAT and GSH-Px, were demonstrated. In addition, the anti-oxidative effect of EGb 761 was positively correlated with the treatment time of EGb 761.

The present study also demonstrated that EGb 761 has a protective effect on MI-induced MSC apoptosis during MSC transplantation. The TUNEL-positive rate in the transplanted MSCs was significantly decreased following EGb 761 treatment, and the TUNEL-positive rate in the transplanted MSCs was negatively correlated with the treatment time of EGb 761.

Apoptosis, or programmed cell death, can be triggered via the extrinsic (death receptor-mediated) signaling pathways. FasL interacts with its receptor, Fas (CD95/APO-1), and then triggers a cascade of subcellular events involved in the extrinsic signaling pathways, which exhibit a role in cell apoptosis (17). In the present study, the level of Fas expression was positively correlated with the time of MI in the control groups. EGb 761 significantly downregulated the expression of the Fas protein and it was negatively correlated with the treatment time of EGb 761. Therefore, the present study hypothesized that the attenuating effect of EGb 761 on cell apoptosis may be mediated by the Fas death receptor signaling pathways. However, the detailed mechanism of the extrinsic apoptotic pathway requires further investigation.

Previously, it has been demonstrated by Daigneault et al (35) that the transplanted MSCs did not express myocyte-specific markers after 2 weeks of transplantation. Our data also demonstrated that MSCs did not express cTnI, a myocyte-specific marker, 7 days after exogenous MSCs were engrafted into infarcted hearts. Therefore, enhancing the differentiation of MSCs into myocytes is a crucial problem in the regeneration of myocardium via stem cell transplantation.

To identify the enhancement of the differentiation of MSCs into cardiac cells, the rats were treated with $100 \mathrm{mg} / \mathrm{kg} /$ day EGb 761 following MSC transplantation. Notably, the present study demonstrated the expression of cTnI in implanted MSCs following 7 days of EGb 761 treatment. However, the detailed mechanisms of the differentiation of MSCs into cardiomyocyte-like cells requires further investigation.

The present study demonstrated the protective effect of EGb 761 on MI following the transplantation of MSCs. These data markedly support the theory that EGb 761 protects implanted-MSCs against MI-induced apoptosis through the activation of the Fas-mediated death receptor signaling pathways. Furthermore, prevention of inflammation, apoptosis and oxidative stress enhanced the differentiation of the transplanted MSCs. The present study provides a promising approach for promoting cardiac repair with a widely available and acceptable therapeutic agent. Therefore, EGb 761 may have considerable significance in improving the efficiency of stem cell therapy for MI. However, due to the complexity of G. biloba composition, further investigation of the detailed mechanisms of specific components involved, particularly in animal ischemic hearts, need to be conducted in order to improve our understanding of the effects of $G$. biloba products.

In conclusion, the present study presents unique evidence that EGb 761 is able to ameliorate the decrease in heart function in a rat model with transplanted MSCs via enhancing the survival and differentiation of implanted MSCs, and attenuating inflammation and oxidative stress in the infarcted myocardial microenvironment.

\section{Acknowledgements}

This study was supported by grants from the National Nature Science Foundation of China (grant no. 31100838; http://www. nsfc.gov.cn).

\section{References}

1. Braunwald E and Bristow MR: Congestive heart failure: fifty years of progress. Circulation 102: IV14-IV23, 2000.

2. Pittenger MF, Mackay AM, Beck SC, Jaiswal RK, Douglas R, Mosca JD, Moorman MA, Simonetti DW, Craig S and Marshak DR: Multilineage potential of adult human mesenchymal stem cells. Science 284: 143-147, 1999.

3. Pereira RF, Halford KW, O'Hara MD, Leeper DB, Sokolov BP, Pollard MD, Bagasra O and Prockop DJ: Cultured adherent cells from marrow can serve as long-lasting precursor cells for bone, cartilage, and lung in irradiated mice. Proc Natl Acad Sci USA 92: 4857-4861, 1995.

4. Schäfer S, Calas AG, Vergouts $M$ and Hermans E: Immunomodulatory influence of bone marrow-derived mesenchymal stem cells on neuroinflammation in astrocyte cultures. J Neuroimmunol 249: 40-48, 2012.

5. Kohyama J, Abe H, Shimazaki T, Koizumi A, Nakashima K, Gojo S, Taga T, Okano H, Hata J and Umezawa A: Brain from bone: efficient 'meta-differentiation' of marrow stroma-derived mature osteoblasts to neurons with Noggin or a demethylating agent. Differentiation 68: 235-244, 2001.

6. Orlic D, Kajstura J, Chimenti S, Jakoniuk I, Anderson SM, Li B, Pickel J, McKay R, Nadal-Ginard B, Bodine DM, Leri A and Anversa P: Bone marrow cells regenerate infracted myocardium. Nature 410: 701-705, 2001. 
7. Liu Y, Yan X, Sun Z, Chen B, Han Q, Li J and Zhao RC: Flk-1+ adipose-derived mesenchymal stem cells differentiate into skeletal muscle satellite cells and ameliorate muscular dystrophy in mdx mice. Stem Cells Dev 16: 695-706, 2007.

8. Makino S, Fukuda K, Miyoshi S, Konishi F, Kodama H, Pan J, Sano M, Takahashi T, Hori S, Abe H, Hata J, Umezawa A and Ogawa S: Cardiomyocytes can be generated from marrow stromal cells in vitro. J Clin Invest 103: 697-705, 1999.

9. Toma C, Pittenger MF, Cahill KS, Byme BJ and Kessler PD: Human mesenchymal stem cells differentiate to a cardiomyocyte phenotype in the adult murine heart. Circulation 105: 93-98, 2002

10. Shake JG, Gruber PJ, Baumgartner WA, Senechal G, Meyers J, Redmond JM, Pittenger MF and Martin BJ: Mesenchymal stem cell implantation in a swine myocardial infarct model: Engraftment and functional effects. Ann Thorac Surg 73: 1919-1925, 2002.

11. Wollert KC, Meyer GP, Lotz J, Ringes-Lichtenberg S, Lippolt P, Breidenbach C, Fichtner S, Korte T, Hornig B, Messinger D, et al: Intracoronary autologous bone-marrow cell transfer after myocardial infarction: the BOOST randomised controlled clinical trial. Lancet 364: 141-148, 2004.

12. Wang Y, Zhang G, Hou Y, Chen J, Wang J, Zou C, Li D, Li H, Zhang Q, Wang A and Fan Q: Transplantation of microencapsulated Schwann cells and mesenchymal stem cells augment angiogenesis and improve heart function. Mol Cell Biochem 366: 139-147, 2012

13. Dong HY, Zhang ZM and Zhou ZX: Effects of endothelin-1 on differentiation of cardiac myocyte induced from rabbit bone marrow stromal cells. Chin Med J (Engl) 119: 832-839, 2006

14. Piao H, Youn TJ, Kwon JS, Kim YH, Bae JW, Bora-Sohn, Kim DW, Cho MC, Lee MM and Park YB: Effects of bone marrow derived mesenchymal stem cells transplantation in acutely infarcting myocardium. Eur J Heart Fail 7: 730-738, 2005.

15. Kamihata H, Matsubara H, Nishiue T, Fujiyama S, Tsutsumi Y, Ozono R, Masaki H, Mori Y, Iba O, Tateishi E, et al: Implantation of bone marrow mononuclear cells into ischemic myocardium enhances collateral perfusion and regional function via side supply of angioblasts, angiogenic ligands, and cytokines. Circulation 104: 1046-1052, 2001.

16. Jacobs BP and Browner WS: Ginkgo biloba: a living fossil Am J Med 108: 341-342, 2000.

17. Yeh YC, Liu TJ, Wang LC, Lee HW, Ting CT, Lee WL, Hung CJ, Wang KY, Lai HC and Lai HC: A standardized extract of Ginkgo biloba suppresses doxorubicin-induced oxidative stress and p53-mediated mitochondrial apoptosis in rat testes. Br J Pharmacol 156: 48-61, 2009.

18. Kang X, Chen J, Xu Z, Li H and Wang B: Protective effects of Ginkgo biloba extract on paraquat-induced apoptosis of PC12 cells. Toxicology in Vitro 21: 1003-1009, 2007.

19. Zhao Z, Liu N, Huang J, Lu PH and Xu XM: Inhibition of cPLA2 activation by Ginkgo biloba extract protects spinal cord neurons from glutamate excitotoxicity and oxidative stress-induced cell death. J Neurochem 116: 1057-1065, 2011.

20. Yang SF, Wu Q, Sun AS, Huang XN and Shi JS: Protective effect and mechanism of Ginkgo biloba leaf extracts for Parkinson disease induced by 1-methyl-4-pheny1-1,2,3,6-tetra-hydropyridine. Acta Pharmacologic Sin 22: 1089-1093, 2001.

21. Cavusoğlu K, Yapar K, Oruç E and Yalçın E: Protective effect of Ginkgo biloba L. leaf extract against glyphosate toxicity in Swiss albino mice. J Med Food 14: 1263-1272, 2011.

22. Wang YC, Zhang Y, Duan AL, Lin WX, Zheng QD and Xu WL: Rapid differentiation of human umbilical cord-derived mesenchymal stem cells into insulin-secreting cells under the sole induction of biological products. Sheng Li Xue Bao 62: 73-78, 2010 (In Chinese).
23. Yoo DY, Nam Y, Kim W, Yoo KY, Park J, Lee CH, Choi JH, Yoon YS, Kim DW, Won MH and Hwang IK: Effects of Ginkgo biloba extract on promotion of neurogenesis in the hippocampal dentate gyrus in C57BL/6 mice. J Vet Med Sci 73: 71-76, 2011.

24. Didier A and Jourdan F: The Ginkgo biloba extract modulates the balance between proliferation and differentiation in the olfactory epithelium of adult mice following bulbectomy. Cell Mol Biol (Noisy-le-grand) 48: 717-723, 2002.

25. Xie XJ, Wang JA, Cao J and Zhang X: Differentiation of bone marrow mesenchymal stem cells induced by myocardial medium under hypoxic conditions. Acta Pharmacol Sin 27: 1153-1158, 2006.

26. Xu SL, Choi RC and Zhu KY: Isorhamnetin, A flavonol aglycone from Ginkgo biloba L, induces neuronal differentiation of cultured PC12 cells: potentiating the effect of nerve growth factor. Evid Based Complement Alternat Med 2012: 278273, 2012.

27. Liu TJ, Yeh YC, Ting CT, Lee WL, Wang LC, Lee HW, Wang KY, Lai HC and Lai HC: Ginkgo biloba extract 761 reduces doxorubicin-induced apoptotic damage in rat hearts and neonatal cardiomyocytes. Cardiovasc Res 80: 227-235, 2008.

28. Liu J, Wang J, Chen X, Guo C, Guo Y and Wang H: Ginkgo biloba extract EGb 761 protects against aging-associated diastolic dysfunction in cardiomyocytes of D-galactose-induced aging rat. Oxid Med Cell Longev 2012: 418748, 2012.

29. Di Nicola M, Carlo-Stella C, Magni M, Milanesi M, Longoni PD, Matteucci P, Grisanti S and Gianni AM: Human bone marrow stromal cells suppress T-lymphocyte proliferation induced by cellular or nonspecific mitogenic stimuli. Blood 99: 3838-3843, 2002.

30. Tse WT, Pendleton JD, Beyer WM, Egalka MC and Guinan EC: Suppression of allogeneic T-cell proliferation by human marrow stromal cells: implications in transplantation. Transplantation 75: 389-397, 2003.

31. Ohnishi S, Yanagawa B, Tanaka K, Miyahara Y, Obata H, Kataoka M, Kodama M, Ishibashi-Ueda H, Kangawa K, Kitamura $\mathrm{S}$ and Nagaya N: Transplantation of mesenchymal stem cells attenuates myocardial injury and dysfunction in a rat model of acute myocarditis. J Mol Cell Cardiol 42: 88-97, 2007.

32. Haines DD, Varga B, Bak I, Juhasz B, Mahmoud FF, Kalantari H, Gesztelyi R, Lekli I, Czompa A and Tosaki A: Summative interaction between astaxanthin, Ginkgo biloba extract (EGb761) and vitamin C in suppression of respiratory inflammation: a comparison with ibuprofen. Phytother Res 1: 128-136, 2011

33. Shi C, Xiao S, Liu J, Guo K, Wu F, Yew DT and Xu J: Ginkgo biloba extract EGb761 protects against aging-associated mitochondrial dysfunction in platelets and hippocampi of SAMP8 mice. Platelets 21: 373-379, 2010.

34. Jiang X, Nie B, Fu S, Hu J, Yin L, Lin L, Wang X, Lu P and Xu XM: EGb761 protects hydrogen peroxide-induced death of spinal cord neurons through inhibition of intracellular ROS production and modulation of apoptotic regulating genes. J Mol Neurosci 38: 103-113, 2009.

35. Daigneault M, De Silva TI, Bewley MA, Preston JA, Marriott HM, Mitchell AM, Mitchell TJ, Read RC, Whyte MK and Dockrell DH: Monocytes regulate the mechanism of T-cell death by inducing Fas-mediated apoptosis during bacterial infection. PLoS Pathog 8: e1002814, 2012. 